\title{
Automated Dynamic and Cooperative Base Station Range Setting in CDMA2000 Cellular Systems
}

\author{
D. N. Balasuriya and S. D. N. A. Jayasooriya
}

\begin{abstract}
Cell shrinking is a common phenomenon in code division multiple access systems in which the cell service area reduces with the loading of the cell. To minimize the service outages resulting from cell shrinking, an automated dynamic and cooperative base station range setting scheme for the CDMA2000 system is proposed. This range setting software resides in a dedicated computer coupled to the back administration module server at the base station controller, periodically checks the traffic reports generated and identifies the loading of cell sectors which in turn initiates the generation of a dynamic range setting script. Automatic execution of the script adjusts the ranges of the neighbouring cell sectors such that the subscribers with high probability of dropping from the loaded sectors due to cell shrinking are now smoothly transferred to their neighbours. This system overcomes most of the practical issues that arise in human controlled manual range changing systems which are currently deployed in Sri Lanka.
\end{abstract}

Keywords: CDMA2000, Cell Shrinking, Service Outages, Dynamic Range Setting

\section{Introduction}

Cellular mobile communication is a rapidly growing industry which allows millions of people around the globe to enjoy the communication facilities while being mobile. Today, it has evolved to a state where the users are bandwidth hungry and are very concerned on quality. The days when a mobile phone was a luxury have passed and the service providers have entered in to a competition to attract subscribers to their networks. Most of the service providers are focusing on service quality to gain an advantage in this competitive cellular mobile market.

From early 90's, Sri Lanka has been served by global system for mobile communication (GSM) networks. However from early 2000, newer technologies like code division multiple access (CDMA), high speed down-link packet access (HSDPA) and very recently long term evolution (LTE) $[1,2]$ have been introduced to Sri Lanka. While embracing the extended capacity improvements via new technologies, in Sri Lanka too, the subscribers are expecting high standards in service quality. Especially in Sri Lanka, the operators and the subscribers are interested in a minimum outage in communication services.

Service outages can occur in multiple ways such as equipment failure, fading or interference. Apart from these, in CDMA cellular mobile systems, cell shrinking $[3,4,5$, 6] is a common phenomenon where the cell radius decreases with the loading of the cell.
This may too cause an outage to the subscribers at the edge of the cell. Specially, this is a severe issue in a Sri Lankan context where one category of CDMA, the CDMA2000 system is deployed to supply fixed telephone services. As the subscriber terminals are not usually mobile in such deployments, they will be experiencing unavoidable outages.

In this paper, an automated dynamic range setting scheme (Mobile Tower Automatic Range Setter - MTARS) is proposed for CDMA2000 cellular mobile systems especially for minimizing the outages caused by the cell shrinking.

The rest of this paper is organized as follows. Section 2 discusses in detail the problem of cell shrinking in a practical CDMA cellular system and the need for a dynamic range setting for minimizing the outages in such a system. In Section 3, the design of the proposed range setting system is presented and in Section 4 , a case study illustrates the operation and the results of the proposed system. Finally a summary and possible future improvements conclude the paper.

Eng. D.N. Balasuriya, AMIE(Sri Lanka), B.Sc. Eng. (Hons)(Moratuwa), MSc(Manitoba), Senior Lecturer, Department of Electrical \& Computer Engineering, The Open University of Sri Lanka.

S.D.N.A. Jayasooriya., BTech (Eng) Undergraduate, Department of Electrical \& Computer Engineering, The Open University of Sri Lanka. 


\section{Cell Shrinking and the Need for Dynamic Range Setting}

CDMA systems code the multiple subscribers' information bit streams with a set of orthogonal codes where one subscriber's coded bit stream sees the other subscribers' bit streams as noise or interference [1]. Hence, once the number of subscribers in the close vicinity of the base transceiver stations (BTS) increases, a subscriber at the edge of the cell observes the other coded bit streams to be adding a considerable noise/interference at the BTS antenna. Once the signal to interference ratio for an edge subscriber falls below the required threshold value, the subscriber mobile is no longer able to communicate with the BTS. However, the subscriber's mobile will always lock to the BTS with the stronger pilot channel $[1,2]$. Hence he will experience a service unreachability while seeing a full signal strength from the BTS.

In most of the CDMA systems, cell breathing [4, $5,6]$ is an available technique to automatically cater for the above issue, in which the pilot channel power of the loaded BTS is gradually reduced such that the subscriber mobiles at the edge of the cell are transferred to the neighbouring cells. For this to be successful, it is very important to have a considerable overlap between the neighbour BTS coverages. In Sri Lanka, most of the operators are focused on minimizing the capital cost in cellular system deployment, hence are interested in deploying the required coverage with the minimum number of BTSes. As a result, the overlap between the coverage areas of neighbour BTSes are at a minimum. In such a situation, configuring the cell breathing is not very fruitful, hence it is not configured.

As mentioned earlier, during the initial planning stage of a cellular network, coverage of a certain cell is determined and set to maintain a balance between the minimum number of BTSes and the maximum possible coverage. Further to this, usually in a practical cellular network, routine radio network optimizations are carried out to identify the long term changes in the subscriber density and to make adjustments to the network so that the network is periodically optimized.

At the same time, unexpected short term subscriber density changes can occur due to localized events such as festivals, musical shows or sports events or even disaster situations. With the increased number of subscribers within the cell's coverage, naturally the CDMA cell's service area will shrink.
However, the neighbouring base stations which are having a lesser subscriber density than the maximum which they can serve are unaware of this sudden change and their range is kept unchanged at the preset optimum values. As the overlap between the neighbour coverages are very less, this reduction of a cell's service area will result in pockets of no service and the subscribers who are in these pockets will see it as an outage or network unreachability. These network unreachabilities may directly affect the subscriber's perception of the network and may result in churning. In a larger scale, this is an enormous loss for a network provider who provides service in a very competitive market. Therefore, most of the service providers are deploying operations staff to manually monitor the subscriber density of the base stations and to dynamically adjust the ranges of base station cells in a trial and error manner. However, this may add errors, delays and most importantly a cost. Most noticeably, the reverting back to the previous optimized network status after the sudden traffic peaks have disappeared is not taken care of well.

Proposed automated dynamic range setting system is capable of identifying the loaded cells and then increasing the coverage of the neighbour cells while decreasing the coverage of the loaded cell such that the subscribers with a high outage probability are smoothly transferred to the neighbour BTSes.

\section{Proposed Automatic Range Setting System}

In this design and implementation of the proposed dynamic BTS range setting system, a widely utilized CDMA2000 system was selected. However, from system to system, the fine details and the implementation of the proposed system may vary. Further, a very important fact to note is that the CDMA2000 BTS cell consists of three sectors which can be range controlled independently. Therefore, from this point onwards we adopt the concept of a sector in place of a cell, but retaining the properties of a cell discussed so far. The design and implementation of the proposed system consists of several sub units (Figure 1).

\subsection{Acquiring the Information for Range Setting}

In the CDMA2000 system, M2000 is a performance monitoring server which queries 


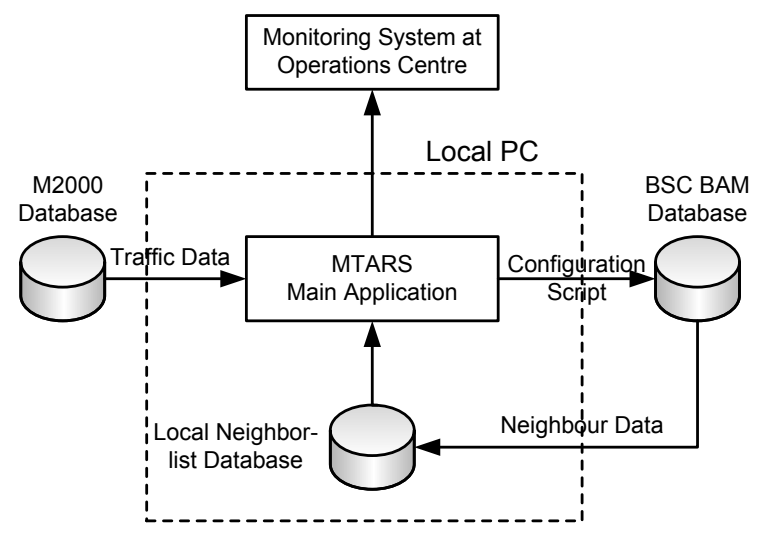

Figure 1- Proposed BTS sector range setter system architecture

the base station controller (BSC) - back administrator module (BAM) databases and extracts traffic data periodically. Then these data are stored in a separate database [8]. One of the main tasks of the proposed system is to query this database and extract the channel element (CE) traffic channel utilization data corresponding to the CDMA2000 base station sectors.

Thereafter, the system filters and selects the BTS sectors with a higher channel utilization than a pre-defined threshold. Note that this threshold is decided at the initial network planning. Furthermore, system maintains a neighbour list database which is periodically synchronized with the BSC-BAM server's neighbour lists. By querying this database, the system identifies the neighbour details corresponding to each loaded BTS sector and the candidate BTS sectors for accepting the dropping subscribers are identified based on their utilization and their geographical coverage area overlap with the loaded BTS sectors. However, the sectors of the same BTS are excluded from the candidate set for handing over the loaded sector traffic in order to avoid unfavourably frequent handoffs.

\subsection{Generating and Executing the Range Changing Script}

According to the data obtained from the BAM server and the M2000 server databases, the handing over of traffic decision is taken as in Figure 2. It is important to note that to avoid delays and errors, we follow a range calculation procedure rather than following a trial and error method practiced in manual range setting.

Before presenting the range calculation, it is important to state the notation used in this calculation.
Let $\lambda_{i j}\left(0<\lambda_{i j} \leq 1\right)$ be the overlap factor between BTS sectors $i$ and $j$ where,

$$
=\frac{\lambda_{i j}}{\text { Area covered both by sector } i \text { and } j}
$$

Further,

$\lambda_{i j_{n} \backslash\left\{j_{1}, j_{2}, \ldots, j_{n-1}\right\}}$

$=\frac{\left[\begin{array}{c}\text { Area covered both by sectors } i \text { and } j_{n} \\ \text { but not covered by sectors } j_{1}, j_{2}, \ldots, j_{n-1}\end{array}\right]}{\text { Area covered by sector } i}$

Note that in the practical deployment of MTARS, these overlap factors are calculated with the available drive test data. Then consider a set of neighbour BTS sectors BTS sector 1, BTS sector $2, . .$, BTS sector $(\mathrm{N})$ with overlap factors of $\lambda_{12}, \lambda_{13 \backslash 2}, \lambda_{14 \backslash\{2,3\}}, \ldots$

\subsubsection{Range Calculation}

Let the upper threshold traffic capacity of a BTS sector is $U$ and the current utilization of BTS sectors are $L_{1}, L_{2}, \ldots, L_{N}$ respectively $\left(L_{1} \geq U>\right.$ $L_{j}$ for $\left.j=2,3,4, \ldots, N\right)$. Let the radius of BTS sector 1 be $R_{0}$. Clearly a traffic transfer from BTS sector 1 is required here to avoid an outage. Let us assume a uniform traffic density in a certain BTS sector coverage area. Then, the traffic volume is proportional to the area of the sector. Furthermore, with the geometric properties of a sector, square root of the area is proportional to the sector radius which inturn results in the square root of traffic volume in a sector being proportional to the sector radius. Thus the new sector radius required to force the sector edge traffic to other BTSes is,

$R=\sqrt{\frac{\alpha U}{L_{1}}} R_{0}$,

where $\alpha(\lesssim 1)$ is a factor selected so that there is a margin kept for further traffic increments in the currently loaded BTS sector. However, in the case of isolated BTSes, in the absence of any candidates for the traffic to be handed over, $\alpha=1$ is selected to avoid unnecessary call drops.

At the same time the new incremented radius of other BTS sectors to capture the dropped traffic can be given as $D_{1 j}-\beta_{j} R$ where $\beta_{j}(<1)$ is a scalar factor which caters for the irregular coverage shapes of the BTS sectors and $D_{1 j}$ is the distance between the two BTSes with sectors 1 and $j$. Further $\beta_{j}$ preserves a coverage overlap region. 


\subsubsection{Remaining CE Traffic Calculation}

This is an iterative candidate selection algorithm where in each iteration, a portion of dropped traffic from BTS sector 1 is handed over to a selected candidate BTS sector. At the end of each iteration, the remaining traffic calculation is twofold depending on the availability of CE capacity at candidates. Let the traffic to be transferred from BTS sector 1 to another BTS sector at the start of a certain iteration be $A$. available CE capacity > Traffic to be transferred):

The transferred traffic to the selected candidate $j$ is $A \lambda_{1 j \backslash\{.\}}$ resulting in a remaining traffic value of $A\left(1-\lambda_{1 j \backslash\{.\}}\right)$.

ii. Traffic calculation $\mathbf{2}$ (When candidate's available CE capacity < Traffic to be transferred):

The transferred traffic to the selected candidate $j$ is $U-L_{j}$ resulting in a remaining traffic value of $A-\left(U-L_{j}\right)$.

i. Traffic calculation $\mathbf{1}$ (When candidate's



Figure 2 - Candidate BTS sector selection and range calculation 
After the BTS sector 1's handover traffic is totally divided between the members of the candidate set, the range changing script is generated by the main application to insert the range changing parameters of the BTS sector 1 and the candidate BTS sectors to BSC-BAM server commands. An example command in the considered CDMA2000 system is shown in Figure 3.

Finally, a file transfer protocol (FTP) session is created between the local computer running the system and the BAM server (which is set to periodically check for the range changing scripts) and the script is transferred via FTP protocol to the BAM [10] (Figure 4). This script is then executed automatically.

\begin{tabular}{|c|c|}
\hline BTS ID = 1 & Sector ID $=0$ \\
\hline BTS Name $=$ KRG & Carrier ID $=9$ \\
\hline Cell ID= 1301 & Metrology $=$ MET \\
\hline Existing Cell radius* & $=40 \mathrm{Km}$ \\
\hline New Cell radius* & $=35 \mathrm{Km}$ \\
\hline Generated command & tring \\
\hline \multicolumn{2}{|c|}{$\begin{array}{l}\text { "SET CBTSCDMA1XSECTORPARA: } \\
\text { BTSID=1, LOCALCELLID=1301, } \\
\text { LOCALSECTORID=0,CRRID=2, } \\
\text { MTRLGY=MET, MAXCELLR=35, } \\
\text { MAXUSRSPD=255, PCM=M25DB, } \\
\text { SAFM=ENABLE;" }\end{array}$} \\
\hline \multicolumn{2}{|c|}{$\begin{array}{l}\text { This command is used to change the existing } \\
\text { BTS sector range } 40 \mathrm{~km} \text { to new range } 35 \mathrm{~km} \text {. }\end{array}$} \\
\hline
\end{tabular}

\section{Figure 3 - Example CDMA2000 BSC command generation}

\subsection{Reverting Back to the Original Range Values}

After setting the ranges of a set of neighbour BTS sectors, MTARS system maintains their previous range values and the set range values in a table. Furthermore, MTARS continues monitoring the traffic in these BTS sectors.
Whenever the traffic of a range reduced BTS sector falls below the pre-defined lower threshold, a new range changing script is generated, transferred to the BAM and executed to revert all the corresponding BTS sector ranges back to the original optimized range values.

There is a possibility that a certain BTS sector range is increased to accept a portion of a loaded neighbour's traffic and later the same BTS sector range is once again changed as a result of a different range setting. During the reverting back process corresponding to previous range change, the BTS sector is always set to its initial optimized range for simplicity. However, due to continuous range setting, any crossing of upper threshold will be detected and the extra load will be distributed eventually.

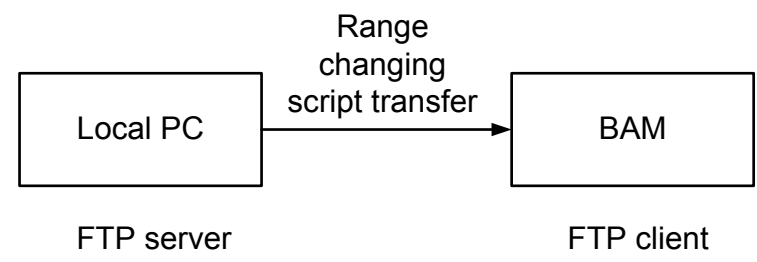

Figure 4 - Script transfer between the local computer and the BAM

\subsection{Monitoring the BTS Sector Range Setting System}

In the proposed system, range setting and reverting back to the original will take place automatically as presented above. However, in a practical CDMA2000 cellular system, it is of highest importance to have a mechanism which allows the operations staff to monitor the changes occurring, when required.

For this, the proposed system provides a monitoring window where all the BTS sectors selected for range setting, their configurations and the candidate neighbour BTS sector details are displayed (Figures 5). The same window can display the recent modifications done, listing the new and previous sector radius values (Figures 6). 


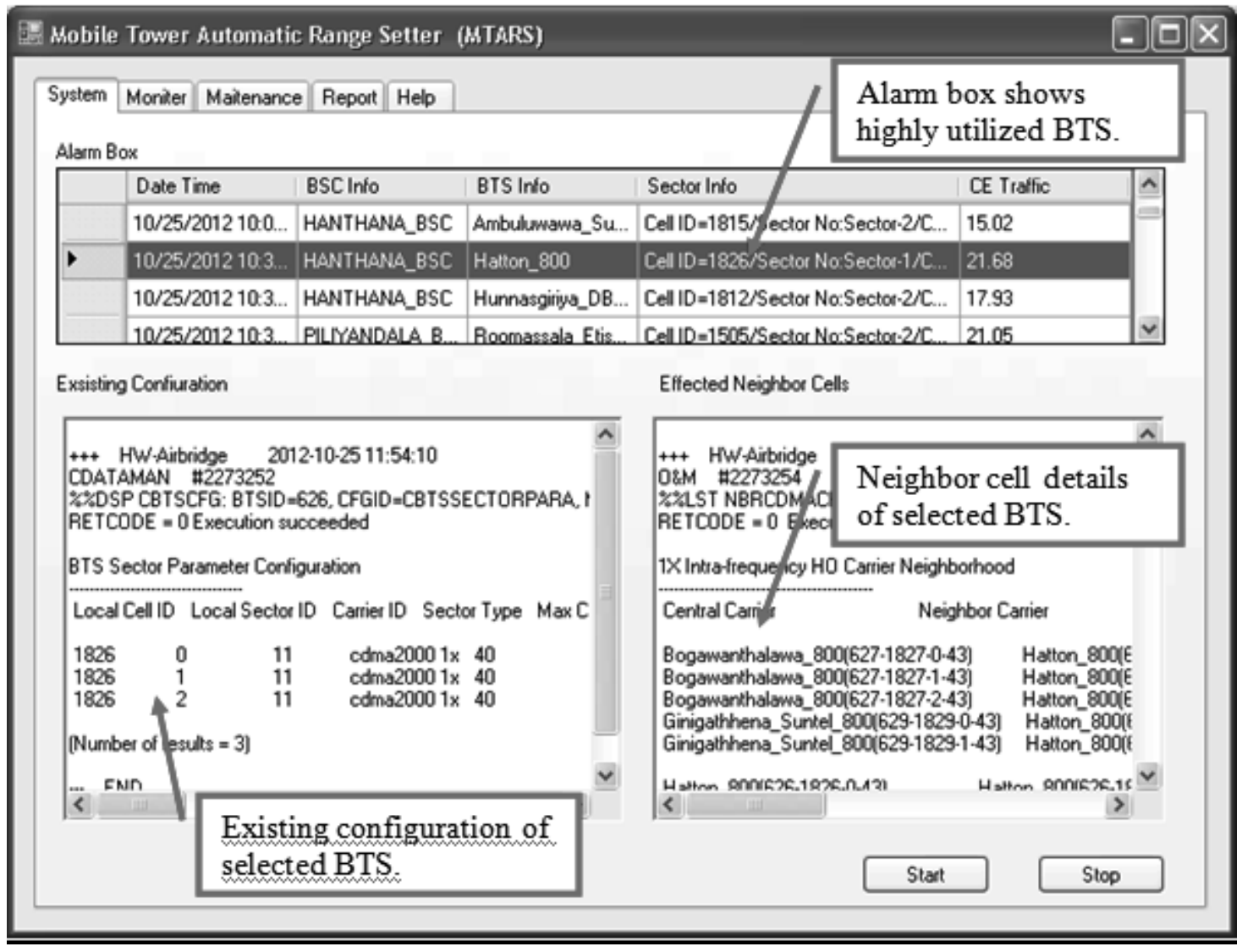

Figure 5 - Monitoring sub-system

F Mobile Tower Automatic Range Setter (MTARS)

0 므

\begin{tabular}{|c|c|c|c|c|c|c|c|c|c|c|}
\hline \multicolumn{4}{|c|}{ Recent Modfication } & \multicolumn{4}{|c|}{$\begin{array}{l}\text { Recent modifications } \\
\text { done through MTARS. }\end{array}$} & \multirow{2}{*}{\multicolumn{2}{|c|}{$\begin{array}{l}\text { Privious } \\
\text { Cell } \\
\text { Radious }\end{array}$}} & \\
\hline & Date Time & BSC Name & $\begin{array}{l}\text { BTS } \\
\text { Name }\end{array}$ & $\begin{array}{l}\text { BTS } \\
\text { ID }\end{array}$ & Cell ID & $\begin{array}{l}\text { Sector } \\
\text { ID }\end{array}$ & $\begin{array}{l}\text { Carries } \\
\text { ID }\end{array}$ & & & \\
\hline & 11/26/2012 11:16 AM & HANTHANA_BSC & PUSSTLLA... & 624 & 1824 & 0 & 11 & 30 & 35 & \\
\hline & 11/26/2012 11:16 AM & HANTHANA_BSC & PUSSELLA... & 624 & 1824 & 0 & 11 & 35 & 40 & \\
\hline & 11/26/2012 11:16 AM & HANTHANA_BSC & PUSSELLA..- & 624 & 1824 & 0 & 11 & 35 & 40 & \\
\hline & $11 / 26 / 2012850 \mathrm{AM}$ & HANTHANA_BSC & Hatton_800 & 626 & 1826 & 1 & 11 & 30 & 35 & \\
\hline & $11 / 26 / 2012850 \mathrm{AM}$ & HANTHANA_BSC & Hatton_800 & 626 & 1826 & 1 & 11 & 35 & 40 & $\checkmark$ \\
\hline
\end{tabular}

Recent Alarm Log

\begin{tabular}{|c|c|c|c|c|c|c|}
\hline & Date Time & BSC Info & BTS Info & rst 10 alarms) & & 1 \\
\hline \multirow[t]{6}{*}{ - } & 11/26/2012 10.00 AM & HANTHANA_BSC & Hatton_80 j & Cel ID=1826/Sector .. & 23.36 & \\
\hline & $11 / 26 / 201210.00 \mathrm{AM}$ & HANTHANA_BSC & Hantha_a_Suntel_800 & Cel ID=1805/Sector ... & 26.44 & \\
\hline & $11 / 26 / 201210.00 \mathrm{AM}$ & HANTHANA_BSC & PUSSELLAWA_800 & Cel ID=1824/Sector ... & 23.3 & \\
\hline & $11 / 26 / 201210.00 \mathrm{AM}$ & HANTHANA_BSC & Arnuradhapuaa_Sunte... & Cel ID=1932/Sector ... & 24.71 & \\
\hline & $11 / 26 / 201210.00 \mathrm{AM}$ & PILYYANDALA_BSC & Roomassala_Etisalat.. & Cel ID $=1505 /$ Sector ... & 24.07 & \\
\hline & 11/26/2012 930 AM & COLOMBO_BSC & Naugala_Suntel_800 & Cel ID=1443/Sector ... & 23.9 & $\checkmark$ \\
\hline
\end{tabular}

Refresh

Figure 6 - Change monitoring window 


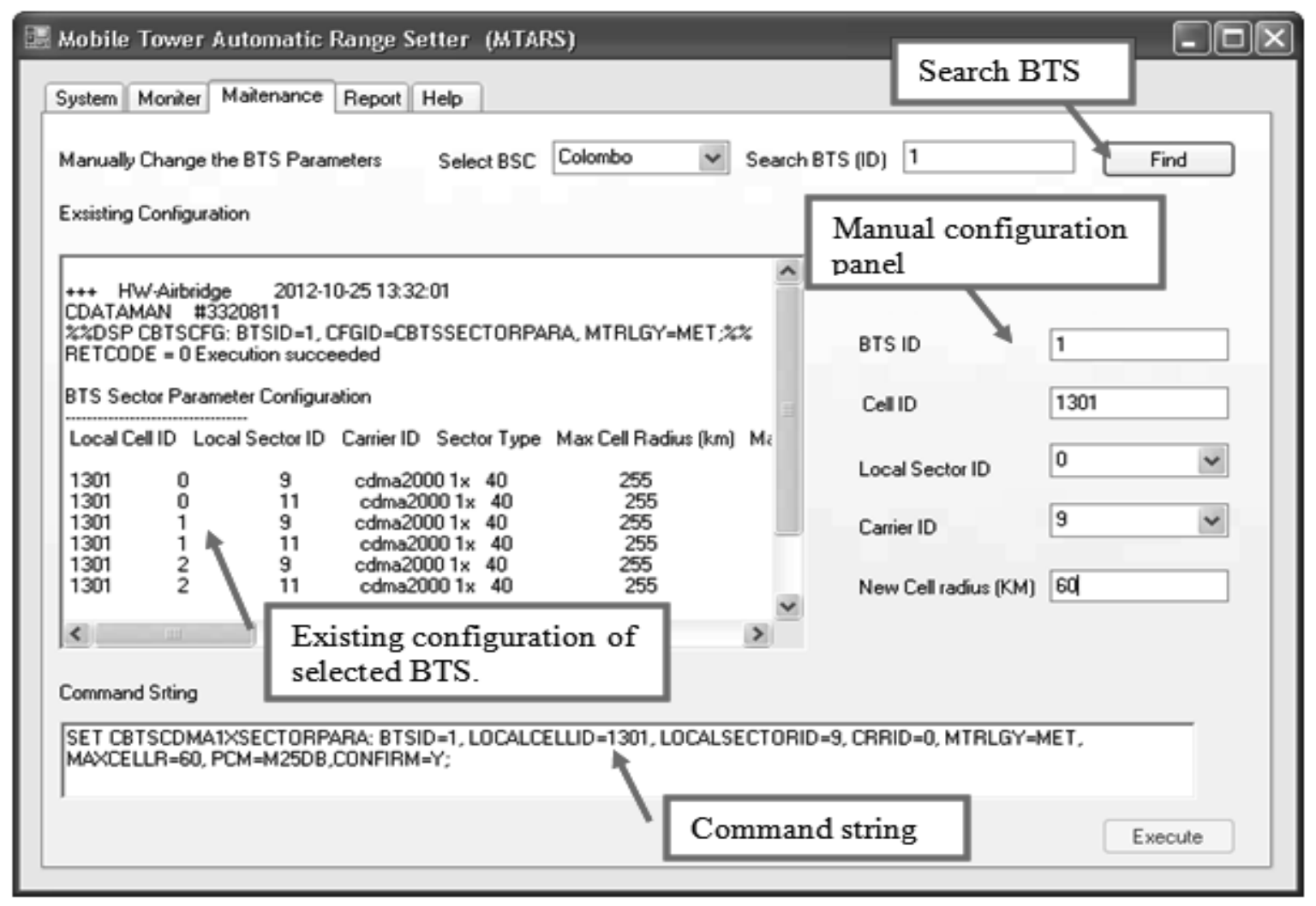

Figure 7 - Manual maintenance window

Furthermore, this extension allows the operations staff to carry out the range setting manually, whenever required (Figure 7). This is an added feature to the proposed system.

\subsection{Software Implementation}

In all these steps, programming language $\mathrm{C} \#$ is used to generate the required classes. One of the important features in this system to note is that the software is created in a modular form so that migrating to an improved system or to a different flat-form is made easier.

\section{- Classes: CSVReader.cs and DBConnector.cs}

Analyse the BTS traffic reports and filter the BTS sectors in which CE Utilizations are above the predefined threshold level. Then send heavily utilized BTS sector data to the network performance alarm indicating table to indicate the BTS sectors' higher utilization.

- Class: MTARS: Form1.cs

Prepares the range changing script.

- Classes: FTPThread.cs and FTPHandler.cs

Transfer the range changing script to the BAM.

\section{A Case Study}

In this section a case study is presented to demonstrate the range setting results after deploying the MTARS in a live CDMA2000 system in Sri Lanka. Here, the upper threshold value was selected as 26 Erlangs and the lower threshold value as 21 Erlangs. Note that these values are solely based on the past experience during manual range setting. Furthermore, for the range calculations $\alpha=\beta=0.9$ is selected. As shown in Figure 8, around 9.00 hours, a peak utilization was observed in a sector of the Pussellawa BTS (BTS sector 1) which was above the upper threshold. At the same time a directly facing, neighbour Watawala BTS sector (BTS sector 2) was having a very less traffic. However, due to the automatic execution of the generated automatic range changing script, it was observed that the traffic was handed-over to BTS sector 2 which resulted in a sudden rise in traffic in BTS sector 2 while BTS sector 1 traffic reduced around 9.30 hours.

Once the traffic of BTS sector 1 has fallen below the lower threshold around 11.30 hours, the reverting back procedure had been automatically executed which resulted in a drop in BTS sector 2 traffic and an increase in BTS sector 1 traffic.

Moreover, the traffic of BTS sector 2 was almost flat during the other day-time hours which 
indicates that no traffic handover was taking place. However, around 20.00 hours there was a night peak in both of the base station sectors, but did not show any traffic handover during the succeeding hour as the threshold was not crossed, which agrees with the range changing algorithms.

\section{Conclusions and Future Work}

An automated dynamic and cooperative BTS sector range setting system has been presented which avoids the practical issues in range setting manually by operations staff. Further, this proposed system allows manual range setting whenever required. A case study presented clearly demonstrates the system implementation results in a real time live CDMA2000 system.

This system can be further improved by adding email and SMS notifications capabilities such that in the case of an automatic BTS sector range change, it is immediately notified to relevant operational teams.

Another future improvement would be to generalize this system to be deployed in any of the CDMA systems irrespective of their vendor and version. This would require standardization of the developed system.

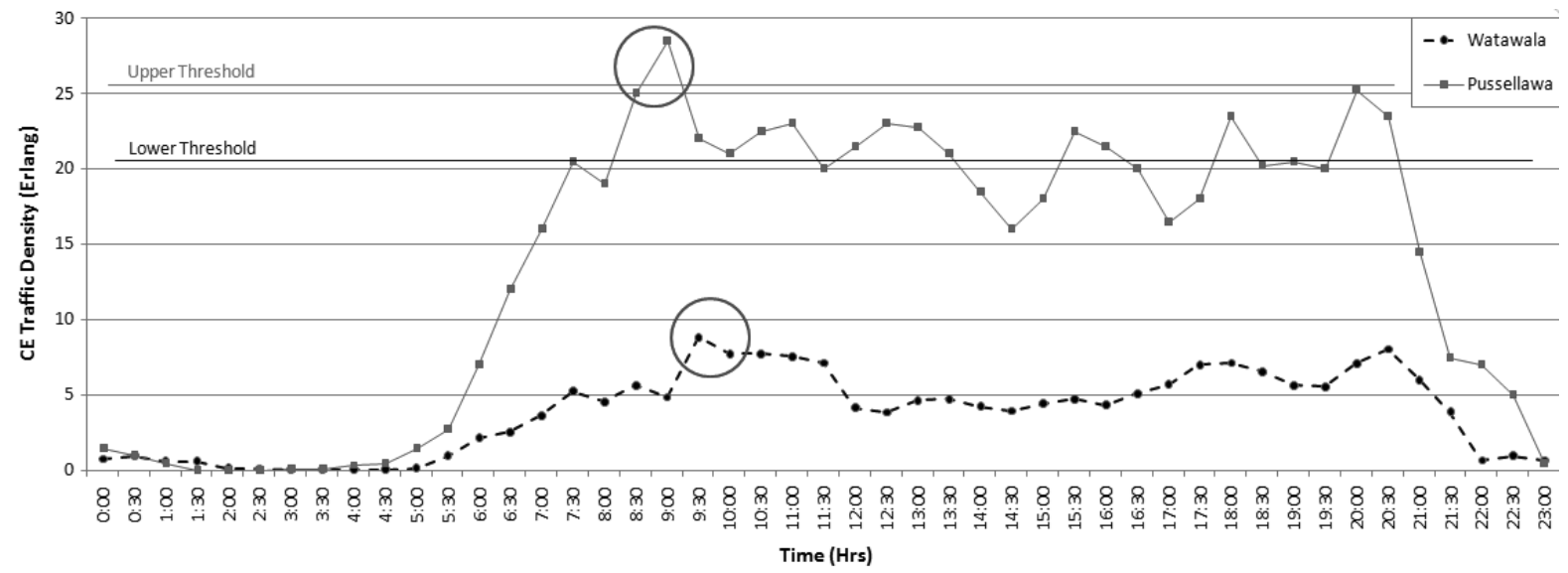

Figure 8 - CE traffic utilization of two facing neighbour BTS sectors at Watawala and Pussellawa BTSes on $26^{\text {th }}$ November 2012

\section{References}

1. Smith C., and Collins, D., 3G Wireless Networks, $1^{\text {st }}$ Ed., McGraw-Hill, 2006.

2. Grag, V., Wireless Network Evolution 2G to 3G, Low Price Edition, Pearson Ed., 2007.

3. Pasaribu, T., "The Developing and Shrinking of a Cell on CDMA Cell because of Increasing Traffic", Jurnal Sistem Teknik Industri, Vol. 7, No. 2, 2006

4. Alani, O., Daoud O., and Abu-Isbeih I., "Coverage Planning in 3G Multimedia Networks Based on Walsh Coding", In Proc. $7^{\text {th }}$ International Multi-Conference on Systems, Signals and Devices, 2010, pp 1-5.

5. Jalali, A., "On Cell Breathing in CDMA Networks", In Proc. IEEE International Conference on Communications, Vol. 2, 1998, pp 985-988.
6. Kelif, J. M., Coupechoux, M., “Cell Breathing, Sectorization and Densification in Cellular Networks", In Proc. $7^{\text {th }}$ International Symposium on Modeling and Optimization in Mobile, Ad Hoc and Wireless Networks, 2009, pp 1-7.

7. Huawei Technologies Ltd., Airbridge cBSC6600 CDMA Base Station Controller Documentation Guide, Ver. T2-032249-20060119-C-1.30, 2006.

8. Huawei Technologies Ltd., CDMA1X Network Optimization Manual, Vol. 1, Issue 1.0, 2006.

9. Huawei Technologies Ltd., HUAWEI UMTS Radio Access Network Documentation Guide, Ver. T2032209-20041001-C-1, 2006.

10. Cliles, A. A., Chiles, D.C. , Manbeck Jr. J. L. , and Nguyen, H., "Techniques for Automatically Updating Software Stored on a Client Computer in a Networked Client-Server Environment", U.S. Patent 6167 567, Dec. 26, 2000. 